

\section{DISCLAIMER}

This report was prepared as an account of work sponsored by an agency of the United States Government. Neither the United States Government nor any agency Thereof, nor any of their employees, makes any warranty, express or implied, or assumes any legal liability or responsibility for the accuracy, completeness, or usefulness of any information, apparatus, product, or process disclosed, or represents that its use would not infringe privately owned rights. Reference herein to any specific commercial product, process, or service by trade name, trademark, manufacturer, or otherwise does not necessarily constitute or imply its endorsement, recommendation, or favoring by the United States Government or any agency thereof. The views and opinions of authors expressed herein do not necessarily state or reflect those of the United States Government or any agency thereof. 


\section{DISCLAIMER}

Portions of this document may be illegible in electronic image products. Images are produced from the best available original document. 
Issued by Sandia National Laboratories, operated for the United States Department of Energy by Sandia Corporation.

NOTICE: This report was prepared as an account of work sponsored by an agency of the United States Government. Neither the United States Government nor any agency thereof. or any of their employees, nor any of their contractors, subcontractors, or their mployees, makes any warranty, express or implied, or assumes any legal liability or esponsibility for the accuracy, completeness, or usefulness of any information, apparatus, product, or process disclosed, or represents that its use would not infringe privately owned rights. Reference herein to any specific commercial product, process, or servic by trade name, trademark, manufacturer, or otherwise, does not necessarily constitute or any agency thereof or any of their

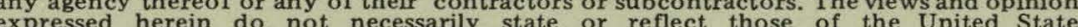
Government, any agency thereof or any of their contractors or subcontractors.

Printed in the United States of America

Available from

National Technical Information Service

U. S. Department of Commerce

5285 Port Royal Road

Springfield, VA 22161

Price: Printed Copy $\$$ '.00; Microtiche: A01 


\section{PAGES 1 to 2 WERE INTENTIONALLY LEFT BLANK}




\title{
MIDTEMPERATURE SOLAR SYSTEMS TEST FACILITY PREDICTIONS FOR THERMAL PERFORMANÇE BASED ON TEST DATA \\ TOLTEC TWO-AXIS TRACKING SOLAR COLLECTOR WITH 3M ACRYLIC POLYESTER FILM REFLECTOR SURFACE
}

Thomas D. Harrison

Experimental Systems Operation Division

Sandia National Laboratories

Albuquerque, NM 87185

\begin{abstract}
Thermal performanse predictions based on test data are presented for the Toltec solar collector, with acrylic film reflector surface, for three output temperatures at five cities in the United States.
\end{abstract}




CONTENTS

Introduction

$\underline{\text { Page }}$

Description of the Collector

Results of the Test Program

Prediction of Thermal Performance 10

$\begin{array}{ll}\text { Previously Published Predictions } & 15\end{array}$

$\begin{array}{ll}\text { Reference } & 17\end{array}$

ILLUSTRATIONS

Figure

1 Toltec Two-Axis Tracking Solar Collector

2 Peak Efficiency Plot for the Toltec Two-Axis Tracking Solar Collector with Acrylic Polyester Film Reflector Surface

3 Thermal Output of the Toltec Two-Axis Tracking Solar Collector with Acrylic Polyester Film Reflector. Surface and Fresno TMY Solar. Data

4 Thermal Output of the Toltec Two-Axis Tracking Solar Collector wilh Acrylic Polyester Film Reflector Surface and Albuquerque TMY Solar Data

5 Thermal Output of the Toltec Two-Axis Tracking Solar Collector with Acrylic Polyester Film Reflector Surface and Fort worth TMY Solar Data

6 Thermal Output of the Toltec Two-Axis Tracking Solar Collector with Acrylic Polyester Film Reflector Surface and Charleston TMY Solar Data 


\section{ILLUSTRATIONS (Continued)}

Figure

7

Thermal Output of the Toltec Two-Axis Tracking Solar Collector with Acrylic Polyester Film Reflector Surface and Boston TMY Solar Data

8 Thermal Output of the Toltec Two-Axis Tracking Solar Collector with Acrylic Polyester Film Reflector Surface and Phoenix TMY Solar Data $\underline{\text { Page }}$

14

14

TABLE

Table

Predicted Annual Thermal Output (kWh/m $\left.{ }^{2} \cdot \mathrm{yr}\right)$ 


\section{MIDTEMPERATURE SOLAR SYSTEMS TEST FACILITY PREDICTIONS FOR THERMAL PERFORMANCE BASED ON TEST DATA \\ TOLTEC TWO-AXIS TRACKING SOLAR COLLECTOR WITH 3M ACRYLIC POLYESTER FILM REFLECTOR SURFACE}

Introduction

Sandia National Laboratories, Albuquerque (SNLA), is currently conducting a program to predict the performance and measure the characteristics of commercially available solar collectors that have the potential for use in industrial process heat and enhanced oil recovery applications. A detailed account of the methods used to make the predictions is given in Reference 1 . For the convenience of the reader, some of this information is repeated in this document. This document presents the thermal performance predictions for the Toltec two-axis tracking solar line-focusing collector. The program is limited to thermal performance only and does not include consideration of other factors, such as

1. Losses at the ends, at gaps, and from shadowing due to packing,

2. Collector warm-up penalties,

3. Degradation of performance,

4. Cost of the collector,

5. Losses in the energy transport system and system warm-up penalties,

6. Reliability,

7. Cost of installation,

8. Cost of operation and maintenance, and

9. Wind effects.

The program is authorized by the Department of Energy, Division of Solar Thermal Energy systems, and is partially funded through the Solar Energy Research Institute. 


\section{Description of the Collector}

A photograph of the Toltec two-axis collector is shown in Figure 1. The module has the following characteristics.

$\begin{array}{ll}\text { Reflector configuration } & \text { Four parabolic troughs } \\ \text { Reflective surface } & 3 \mathrm{M} \text { acrylic polyester film } \\ \text { Aperture dimensions } & 1.02 \mathrm{~m} \times 2.95 \mathrm{~m}(3.35 \mathrm{ft} \times 9.68 \mathrm{ft}) \\ \text { Aperture area (four troughs) } & 12.04 \mathrm{~m}^{2}\left(129.6 \mathrm{ft}^{2}\right) \\ \text { Support structure } & \text { Fiberglass on a steel frame } \\ \text { Tracking system } & \text { Shadow band, two-axis tracking } \\ \text { Deive mechanism } & \text { Electric } \\ \text { Heat transfer fluid } & \text { Therminol } 66^{\circ} \\ \text { Operating range } & 50^{\circ} \text { to } 200^{\circ} \mathrm{C}\left(112^{\circ} \text { to } 392^{\circ} \mathrm{F}\right) \\ \text { Manufacturer } & \text { Toltec, Inc. } \\ & 40 \text { th and East Main, RR/1 } \\ & \text { Clear Lake, IA } 50428\end{array}$

Results of the Test Program

This collector was tested at Sandia National Laburatories, Albuquerque, New Mexico. From the test data, two parameters were defined.

1. Peak efficiency ( $\left.n_{p}\right)$-- the efficiency of the collector when the sun's rays are at normal incidence to the aperture plane (equivalent to solar noon) expressed as a function of $\Delta t / I$. $\Delta t$ is lile temperature difference between the heat transfer fluid outlet temperature and the ambient temperature in degrees centigrade. I is the irradiance of the sun in watts per square meter.

$$
n_{p}=72.2-83.6 \Delta t / 1-62.51(\Delta t / 1)^{2} .
$$

Peak efficiency is plotted in Figure 2. 


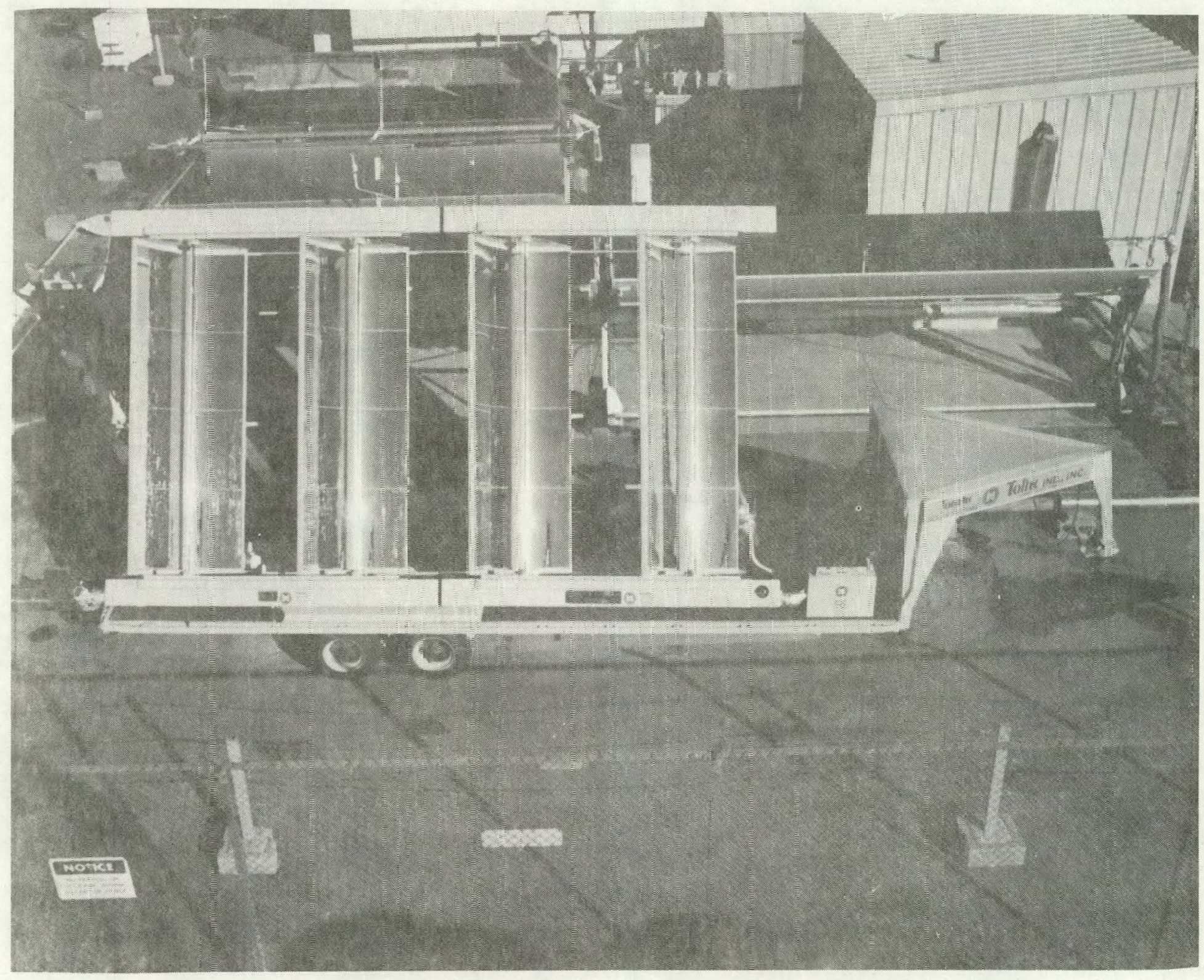

Figure 1. To-tec Two-Axis Tracking Solar Collector 


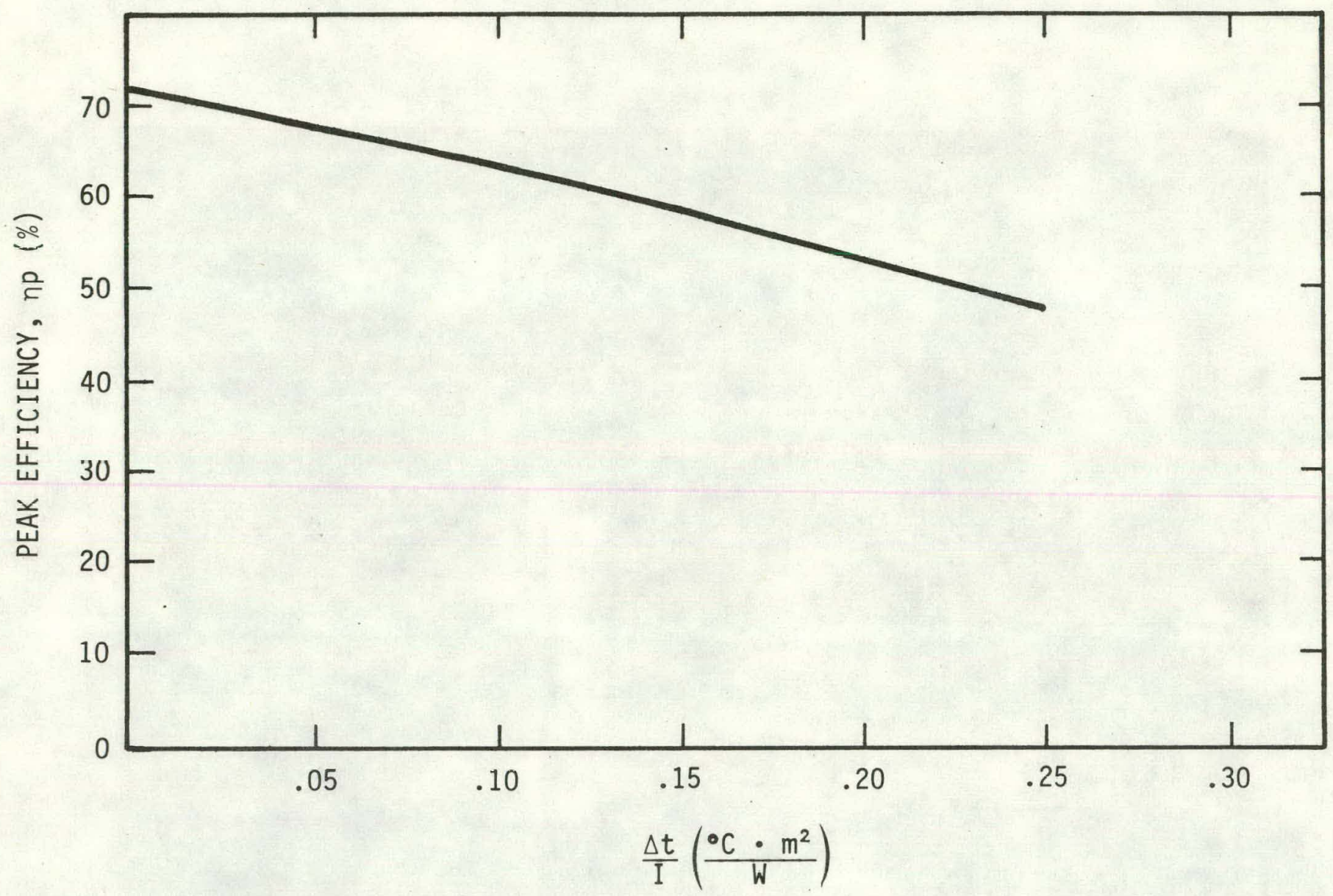

Figure 2. Peak Efficienry Plot for the Toltec

Two-Axis Tracking Solar Collector with

Acrylic Polyester Film Reflector Surface

2. Optical losses -- The value of the optical incident angle modifier for two-axis tracking collectors is judged to be 1 at all times because the incident angle is always zero degrees.

\section{Prediction of Thermal Performance}

A computer program calculates the predicted thermal performance of the collector. The performance parameters defined above are the input data describing the collector while solar and weather data are provided by TMY data tapes. With this input, the computer program calculates 
the thermal output of the collector for each month of the TMY in units of kilowatt hours per square meter $\left(\mathrm{kWh} / \mathrm{m}^{2}\right)$ of collector aperture area. This calculation was made for six locations: Fresno, California; Albuquerque, New Mexico; Fort Worth, Texas; Charleston, South Carolina; Boston, Massachusetts; and Phoenix, Arizona. Three different collector output temperatures were considered. Figures 3 through 8 are graphical displays of the results of the computer prediction. These figures show the monthly thermal output $\left(\mathrm{kWh} / \mathrm{m}^{2} \cdot \mathrm{mo}\right)$ for each location and output temperature. The monthly outputs have been summed to give the annual output for each parameter variation, and the results are shown in Table 1. The computer predictions assume' l square meter of collector aperture in the middle of a row of infinite length, with no end or gap losses and no shadowing due to packing. 


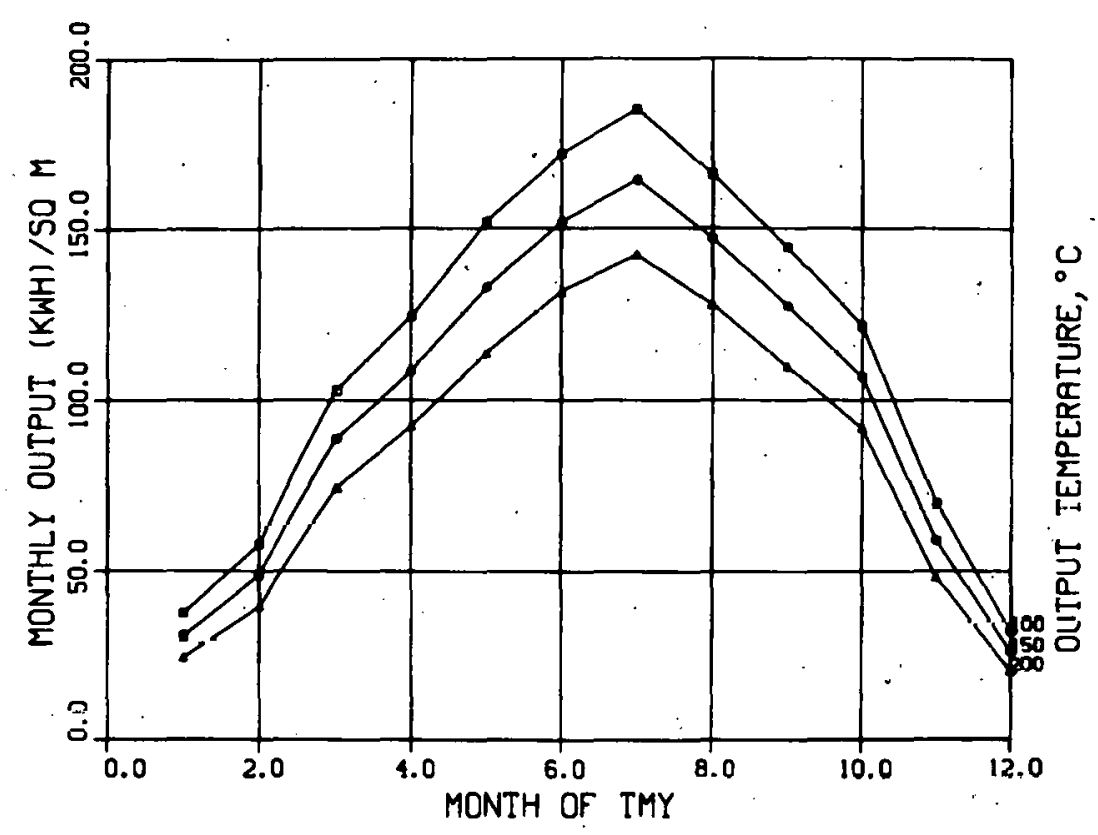

Figure 3. Thermal output of the Toltec Two-Axis Tracking Solar Collector with Acrylic Polyester Film Refléctor Surface and Fresno TMY Solar Data.

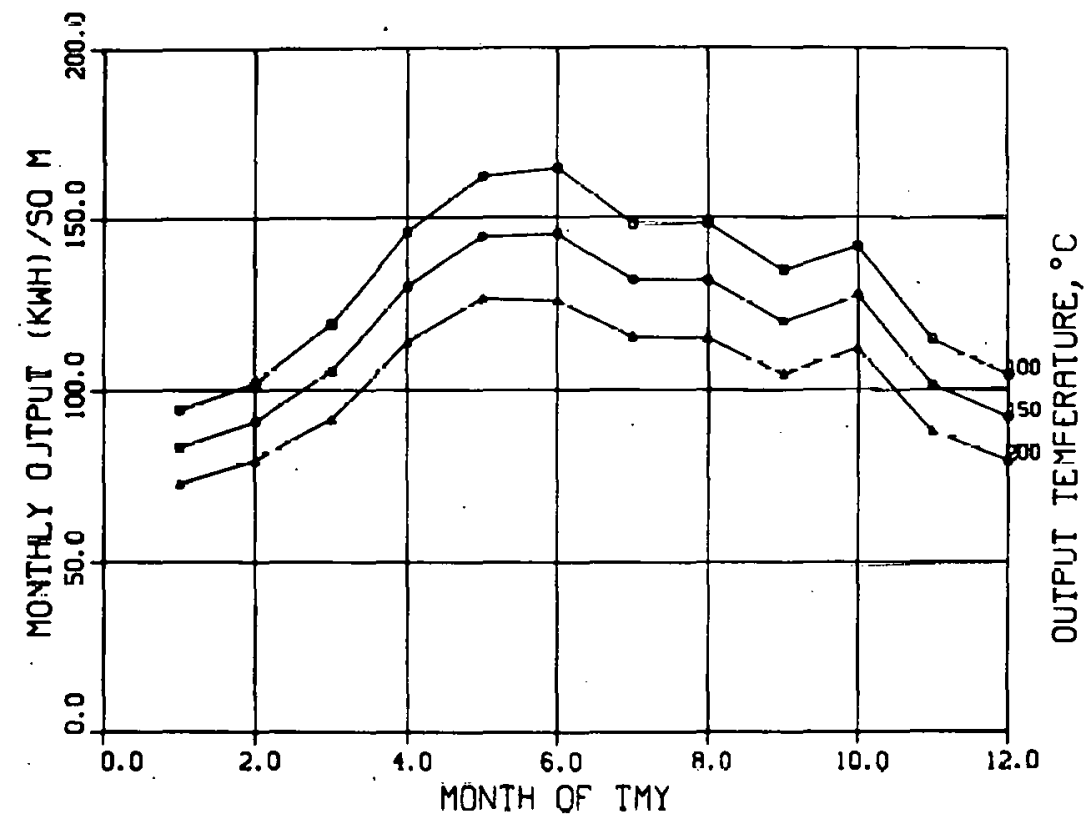

Figure 4. Thermal Output of the Toltec Two-Axis Tracking Solar Collector with Acrylic polyester Film Reflector Surface and. Albuquerque TMY Solar Data 


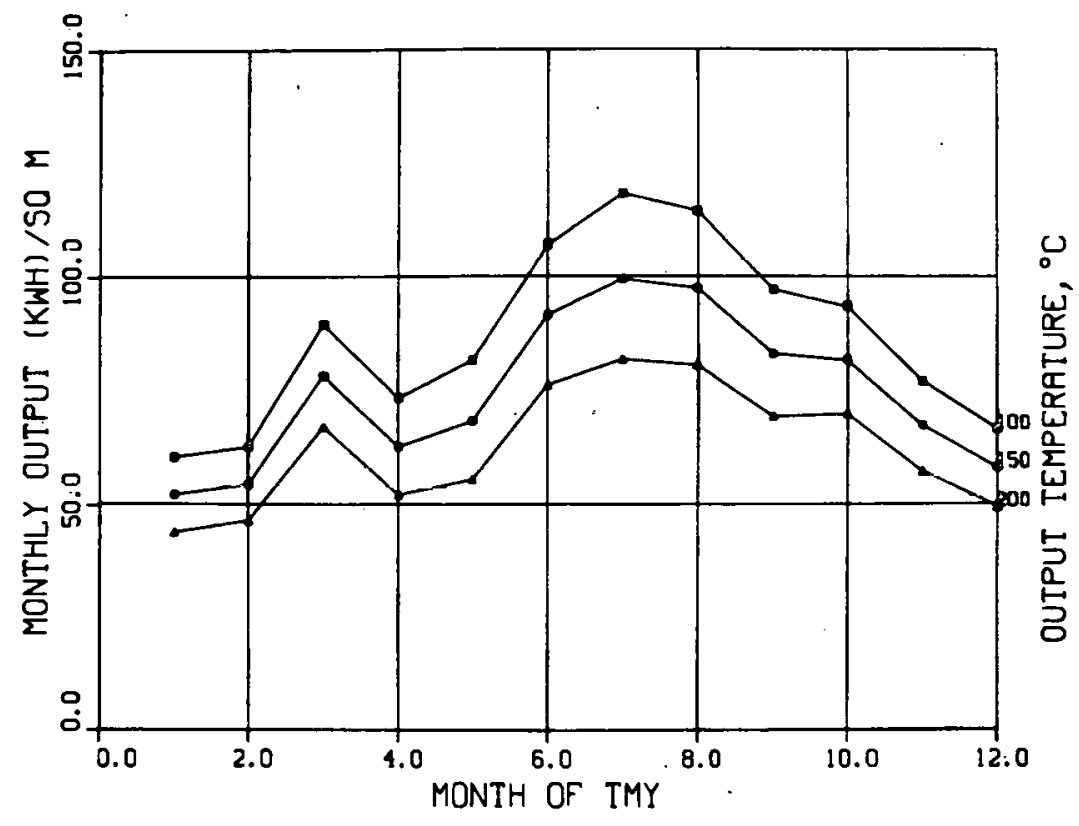

Figure 5. Thermal output of the Toltec Two-Axis Tracking Solar Collector with Acrylic Polyester Film Reflector Surface and Fort Worth TMY Solar Data

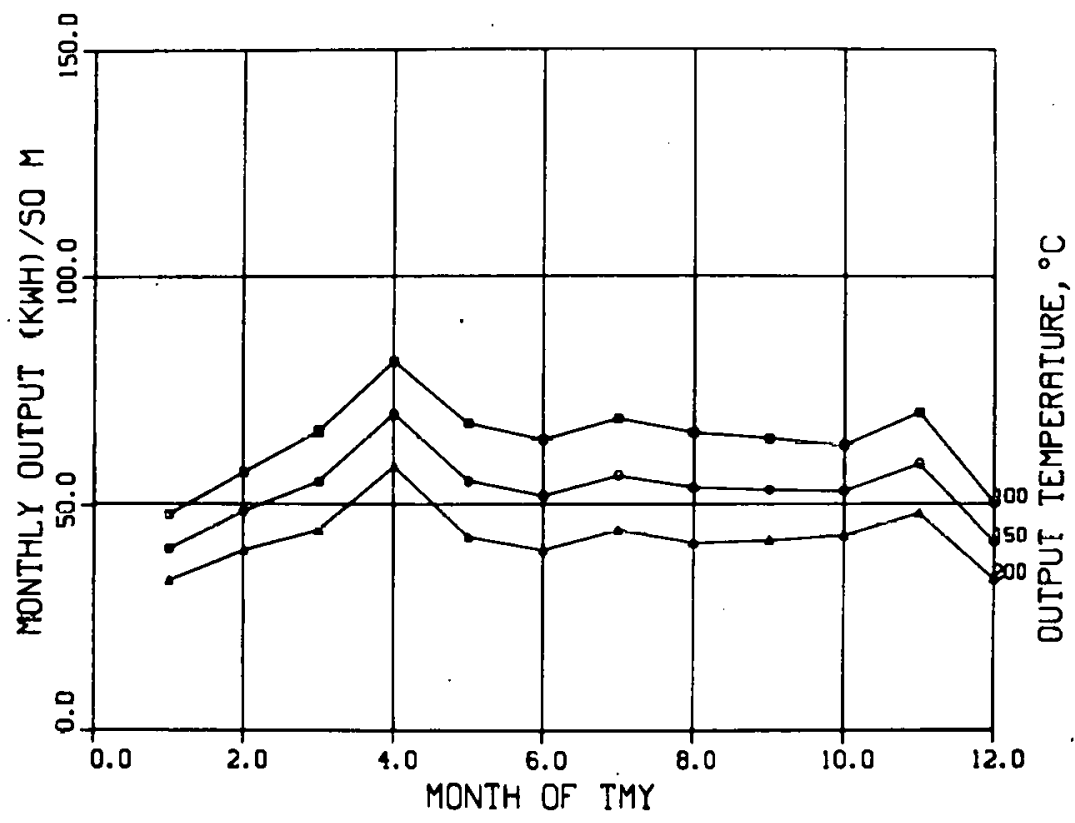

Figure 6. Thermal Output of the Toitec Two-Axis Tracking Solar collertor with Acrylic Polyester Film Reflector surface and Charleston TMY Solar Data 


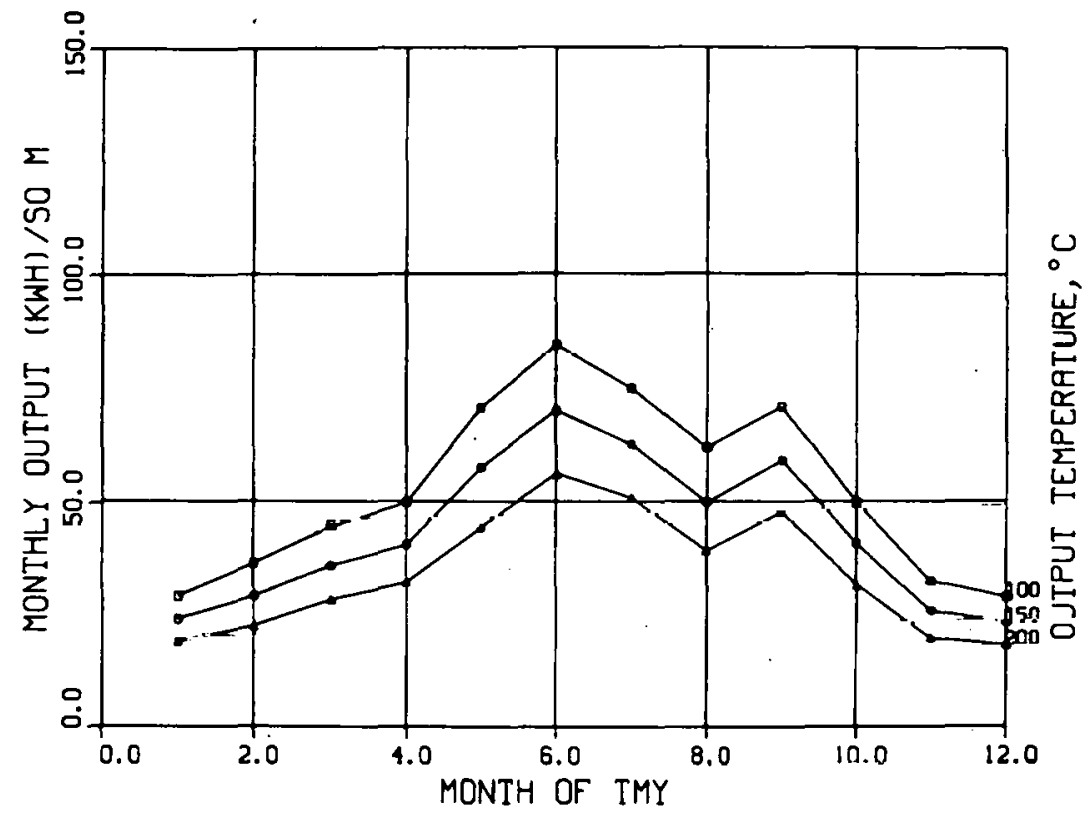

Figure 7. Thermal output of the Toltec Two-Axis Tracking Solar collector with Acrylic Polyester Film Reflector Surface and Boston TMY Solar Data

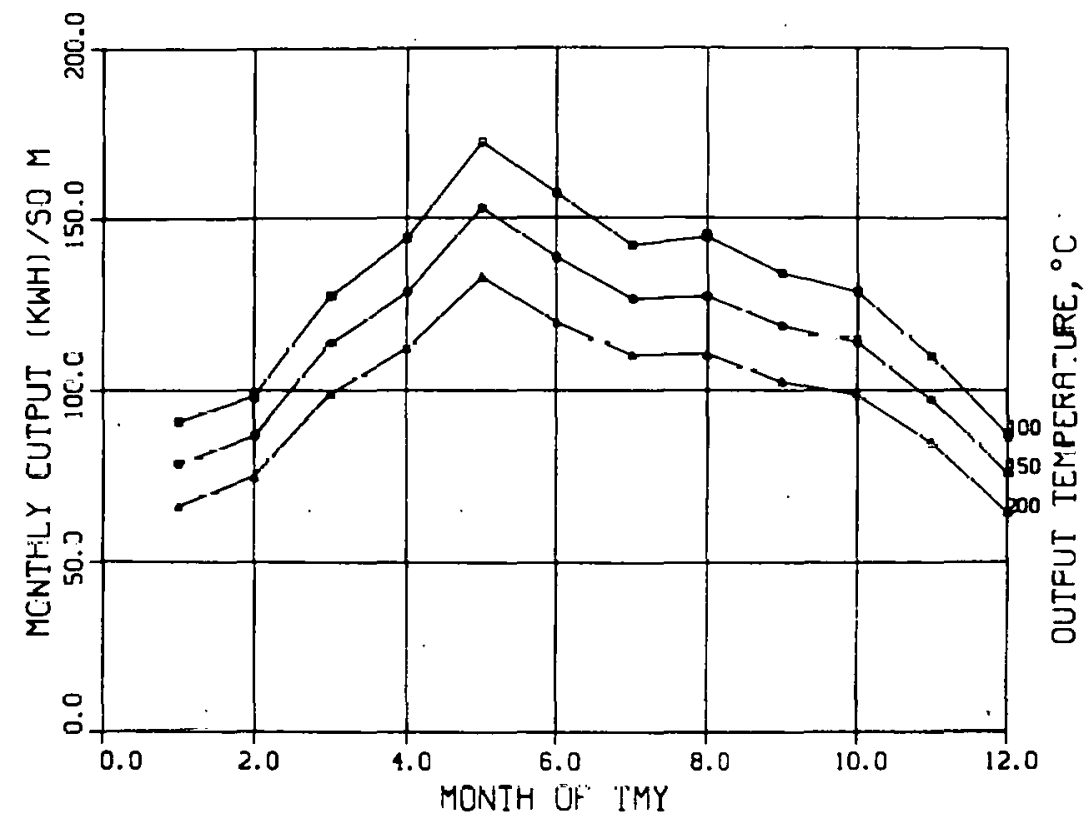

Figure 8. Thermal output of the Toltec Two-Axis Tracking Solar Collector with Acrylic Polyester Film Reflector Surface and Phocnix TMY Solar Data 
Table 1

Predicted Annual Thermal Output (kWh $\left./ \mathrm{m}^{2} \cdot \mathrm{yr}\right)$

\section{Solar Energy Available}

Fresno

Albuquerque

Fort worth

Charleston

Boston

Phoenix
2260

2583

1764

1358

1173

2477
Output Temperature

\begin{tabular}{rrr}
\hline $100^{\circ} \mathrm{C}$ & $\frac{150^{\circ} \mathrm{C}}{1193}$ & $\frac{200^{\circ} \mathrm{C}}{1018}$ \\
1366 & 1403 & 1224 \\
1579 & 894 & 750 \\
1041 & 638 & 514 \\
766 & 520 & 412 \\
635 & 1358 & 1176 \\
1536 & &
\end{tabular}

CAUTION: The above predictions are somewhat optimistic for more than one trough. Thermal losses from the manifolds which interconnect the four troughs were included in the calculation of the efficiency equation, and there were no end losses because the incident angle is zero degrees. However, as stated on page 10 of Reference 1, losses due to shadowing were not included. For low sun angles, there is some shadowing of troughs behind the one nearest the sun. The amount of shadowing is a function of latitude, season of the year, and trough spacing.

This is the final report in this series.

\section{Previously Published Predictions}

Thomas D. Harrison, Midtemperature Solar Systems Test Facility Predictions for Thermal Performance of the Solar Kinetics T-700 Solar Collector with FEK 244 Reflector Surface, SAND80-1964/1 (Albuquerque: Sandia National Laboratories, November 1980). 
Thomas D. Harrison, Midtemperature Solar Systems Test Facility Predictions for Thermal Performance of the Suntec Solar Collector with Heat-Formed Glass Reflector Surface, SAND80-1964/2 (Albuquerque: Sandia National Laboratories, November 1980).

Thomas D. Harrison, Midtemperature Solar Systems Test Facility Predictions for Thermal Performance of the Acurex Solar Collector with FEK 244 Reflector Surface, SAND80-1964/3 (Albuquerque: Sandia National Laboratories, January 1981).

Thomas D. Harrison, Midtemperature Solar Systems Test Facility Predictions for Thermal Performance Based on Test Data; Sun-Heet Nontracking Solar Collector, SAND80-1964/4 (Albuquerque: Sandia National Laboratories, March 1980).

Thomas D. Harrison, Midtemperature Solar Systems Test Facility Predictions for Thermal Performance Based on Test Data; AAI Solar Collector with Pressure-Formed Glass Reflector Surface, SAND80-1964/5 (Albuquerque: Sandia National Laboratories, March 1981).

Thomas D. Harrison, Midtemperature Solar Systems Test Facility Predictions for Thermal Performance Based on Test Data; Acurex Solar Collector with Glass Reflector Surface, SAND80-1964/6 (Albuquerque: Sandia National Laboratories, March 1981).

Thomas D. Harrison, Midtemperature Solar Systems Test Facility Predictions for Thermal Performance Based on Test Data; Solar Kinetics T-700 Solar Collector with Glass Reflector Surface, SAND80-1964/7 (Albuquerque: Sandia National Laboratories, March 1981).

Thomas D. Harrison, Midtemperature Solar Systems Test Facility Predictions for Thermal Performance Based on Test Data; Alpha Solarco Model 104 Solar Collector with 0.125 Low-Iron Glass Reflector Surface, SAND80-1964/8 (Albuquerque: Sandia National Laboratories, April 1981) .

Thomas D. Harrison, Midtemperature Solar Systems Test Facility Predictions for Thermal Performance Based on Test Data; Solar Kinetics T-600 Solar Collector with FEK 244 Reflector Surface, SAND80-1964/9 (Albuquerque: Sandia National Laboratories, April 1981). 
Thomas D. Harrison, Midtemperature Solar Systems Test Facility Predictions for Thermal Performance Based on Test Data; Custom Engineering Solar Collector with Glass Reflector surface, SAND80-1964/10 (Albuquerque: Sandia National Laboratories, April $1981)$.

Thomas D. Harrison, Midtemperature Solar systems Test Facility Predictions for Thermal Performance Based on Test Data; Polisolar Model POL Solar Collector with Glass Reflector Surface, SAND80-1964/11 (Albuquerque: Sandia National Laboratories, May 1981).

\section{Reference}

${ }^{1}$ T. D. Harrison, Midtemperature Solar Systems Test Facility Program for predicting Thermal Performance of Line-Focusing, Concentrating Solar Collectors, SAND80-1964 (Albuquerque: Sandia National Laboratories, November $\left.1980^{\circ}\right)$. 


\section{DISTRIBUTION :}

TID-4500-R66, UC62 (.268), 11/80

AAI Corporation

P.O. Box 6787

Baltimore, MD 21204

Acurex Aerotherm

485 Clyde Avenue

Mountain View, CA 94042

Attn: J. Vindum

Advanco Lorpōio tion

999 N. Sepulvera Rlva.

Suite 314

El Segundo, CA 90245

Attn: B. J. Washom

Alpha Solarco

1014 Vine Street

Suite 2230

Cincinnati, OH 45202

American Boa, Inc.

Suite 4907, One World

Trade Center

New York, NY 10048

Attn: R. Brundage

Anaconda Metal Hose Co.

698 South Main Street

Waterbury, Cr 06720

Attn: $W$. Genshino

Applied Concepts Corp.

P.O. Box 2760

Reston, VA 20090

Attn: J. S. Hauger

Applied Solar Resources, Inc. 490 East Pima

Phoenix, AZ 85004

Attn: W. H. Coady

Arizona Public Service Co.

Bux 21666 MS 1795

Phoenix, AZ 85036

Attn: Dr. B. L. Broussard
Argonne National Laboratory (3)

9700 South Cass Avenue

Argonne, IL 60439

Attn: K. Reed

W. W. Schertz

R. Winston

BDM Corporation

1001 Randolph Etrcet

Albuquerque, NM 87106

Attn: T. Reynolds

Battelle Memorial Institute

Pacific Northwest Laboratory

P.O. Box 999

Richland, WA 99352

Attn: K. Drumheller

Bechtel National, Inc.

P.O. Box 3965

50 Beale Street

San Francisco, CA 94119

Attn: E. Y. Lam

Black and veatch (2)

P.O. Box 8405

Kansas City, MO 64114

Attn: Dr. J. C. Grosskreutz

D. C. Gray.

Boeing Space Center (2)

$M / S$ 86-0I

Kent, W.A 98131

Attn: S. Duzick

A. Lunde

Boomer-Fiske, Inc.

4000 S. Princeton

Chicago, IL 60609

Attn: C. Cain

Budd Company

Fort Washington, PA 1.9034

Attn: W. W. Dickhart

The Budd Company

Plastic R\&D Center

356 Executive Drive

Troy, MI 48084

Attn: J. N. Epel 
DISTRIBUTION (Continued)

Carrier Corp.

Energy Systems Div.

Summit Landing

P.O. Box 4895

Syracuse, NY 13221

Attn: R. A. English

Compudrive Corp.

76 Treble Core Road

N. Billerica, MA 01862

Attn: T. Black

Cone Drive

Division of Excello Corp.

P.O. Box 272

240 E. 12 St.

Traverse City, MI 49684

Attn: J. E. McGuire

Congressional Research Service

Library of Congress

Washington, DC 20540

Attn: H. Bullis

Corning Glass Co. (2)

Corning, NY 14830

Attn: A. F. Shoemaker

W. Baldwin

Custom Engineering, Inc.

$2805 \mathrm{~S}$. Tejon St.

Englewood, CO 80110

Attn: C. A. demoraes

DSET

Black Canyon Stage

P.O. Box 185

Phoenix, AZ 85029

Attn: G. A. Zerlaut

Del Manufacturing Co.

905 Monterey Pass Road

Monterey Park, r.A 91754

Attn: M. M. Delgado

Desert Research Institute

Energy Systems Laboratory

1500 Buchanan Blva.

Boulder City, NV 89005

Attn: J. O. Bradley
Donnelly Mirrors, Inc.

49 West Third Street

Holland, MI 49423

Attn: J. A. Knister

E-Systems, Inc.,

Energy Tech. Center

P.O. Box 2.26118

Dallas, TX 75266

Attn: R. R. Walters

Easton Utilities Commission

219 North Washington St.

Easton, MD 21601

Attn: Mr. W. H. Corkran, Jr.

Eaton Corporation

Industrial Drives Operations.

Cleveland Division

3249 East $80 \mathrm{st}$.

Cleveland, OH 44104

Attn: R. Glatt

Edison Electric Institute

90 Park Avenue

New York, NY 10016

Attn: L. O. Elsaesser

Electric Power Research

Institute (2)

3412 Hillview Avenue

Palo Alto, CA 94303

Attn: Dr. J. Cummings

$$
\text { J. E. Bigger }
$$

Energetics

833 E. Arapahoe street

Suite 202

Richardson, TX 75081

Attn: G. Bond

Energy Institute

1700 Las Lomas NE

Albuquerque, NM 87131

Eurodrive, Inc.

2001 W. Main. St.

Troy, OH 45373

Attn: S. D. Warner 
DISTRIBUTION (Continued)

Exxon Enterprises (3)

P.O. Box 592

Florham Park; NJ 07923

Attn: J. Hamilton

P. JoY

Dr. M. C. Noland

Florida Solar Energy Center (2)

300 State Road, Suite 401

Cape Canaveral, FL 32920

Attn: C. Beech

D. Block

Ford Aerospace and Communications

3939 Fabian Way

Palo Alto, CA 94303

Attn: H. J. Sund

Ford Glass Division

Glass Technical Center

25500 West Outer Drive

Lincoln Park, MI 48146

Attn: H. A. Hill

General Atomic

P.O. Box 81608

San Diego, CA 92138

At.t.n: A. Schwartx

General rilectric Co. (2)

P.O. BOX 8661

Fhilaulelphiá, HA 19101

Attn: $W$. Pijawka

C. Billingsley

General Motors

Harrison Radiator Division

Bldg. 6, Dept. $\cap \cap 3$

Lockport, NY 14094

Attn: L. Brock

General Mutors Corporation

Technical Center

Warren, MI 48090

Attn: J.F. Britt

Georgia Institute of Tcchnology

Atlanta, GA 30332

Attn: J. D. Walton
Georgia Power Company

270 Peachtree

P.O. Box 4545

Atlanta, GA 30302

Attn: J. Roberts

Glitsch, Inc.

P.O. Box 226227

Dallas, TX 75266

Attn: R. W. MCClain

Haveg Industries, Inc.

1287 E. Imperial Highway

Santa Fe Spririgs, CA 90670

Attn: J. Flynt

Hexcel

11711 Dublin Blvd.

Dublin, CA 94566

Attn: R. Johnston

Highland Plating

1128 N. Highland

Los Angeles, CA 90038

Attn: M. Faeth

Honeywell, Ins.

Energy Resources center

2600 Ridgeway Parkway

Minncapolis, MN 55413

Attn: J. R. Willidlls

Insights West

900 Wilshire Blvd.

Los Angeles, CA 90017

Attn: J. H. williams

Jacobs Engineering Co. (2)

251 South Lake Avenue

Pasadena, CA 91101

Attn: B. Eldridge

R. Morton

Jet Propulsion Laboratory (3)

4800 Oak Grove Drive

Pasadena, CA 91103

Attn: J. Becker

J. Lucas

V. C. Truscello 
DISTRIBUTION (Continued)

Kingston Industries Corporation 205 Lexington Ave.

New York, NY 10016

Lawrence Livermore Laboratory University of California P.O. Box 808

Livermore, CA 94500

Attn: W. C. Dickinson

Los Alamos National Lab. (3)

Los Alamos, NM 87545

Attn: J. D. Balcomb

C. D. Bankston

D. P. Grimmer

McDonnell Douglas

Astronautics Company (3)

5301 Bolsa Avenue

Huntington Beach, CA 92647

Attn: J. B. Blackmon

J. Rogan

D. Steinmeyer

Morse Chain

Division of Borg-Warner Corp.

4650 Steele St.

Denver, CO 80211

Attn: G. Fukayama

Motorola Inc.

Government Electronics Division

8201 E. McDowell Road

P.O. Box 1417

Scottsdale, A's 85252

Attn: R. Kendall

New Mexico State University

Solar Energy Department

Las Cruces, NM 88001

Oak Ridge National Laboratory (3)

P.O. BoX Y

Oak Ridge, 'IN 37830

Attn: S. I. Kaplan

G. Lawson

W. R. Mixon

Office of Technology Assessment U.S. Congress

Washington, DC 20510

Attn: R. Rowberg
Omnium G

1815 Orangethorpe Park

Anaheim, CA 92801

Attn: S. P. Lazzara

Owens-Ill inois

$1020 \mathrm{~N}$. Westwood

Toledo,.OH 43614

Attn: Y. K. Pei

PPG Industries, Inc.

One Gateway Center

Pittsburgh, PA 15222

Attn: C. R. Frownfelter

PRC Energy Analysis Company

7600 old Springhouse Road

McLean, VA 22102

Attn: J. Meglan

Parsons of California

3437 S. Airport Way

Stockton, CA 95206

Attn: D. R. Biddle

Progress Industries, Inc.

7290 Murdy Circle

Huntington Beach, CA 92647

Attn: K. Busche

Ronel Technetics, Inc:

501 West Sheridan Rd.

McHenry, IL 60050

Attn: N. Wensel

Schot.t America

11 East 26th St.

New York, NY 10010

Attn: J. Schrauth

Scientific Applications, Inc. 100 Mercantile, Commerce Bldg. Dallas, $\mathrm{TX} 75201$

Aten: Dr. J. W. Doane

Scientific Atlanta, Inc. 3845 Pleasantdale Road

Atlanta, GA 30340

Attn: A. Ferguson 


\section{DISTRIBUTION (Continued)}

Solar Energy Information Center 1536 Cole Blvd. Golden, CO 80401

Attn: R. Ortiz

Solar Energy Research

Institute (113)

1536 Cole Blvd.

Golden, $C O 80401$

Attn: B. L. Butler

L. G. Dunham (4)

B. P. Gupta

F. Kreith

U. 'Thornton

K. Touryan

N. Woodley

D. W. Kearney

C. Bishop

B. Feasby

A. Lewandowski (100)

Solar Energy Technology Rocketdyne Division 6633 Canoga Avenue

Canoga Park, CA 91304

Attn: J. M. Friefeld

Solar Kinetics Inc.

P.O. Box 47045

8120 Chancellor Row

Dallas, TX 75247

Attn: G. Hutchinson

Southwest Research Institute

P.O. Bux 28510

San Antonio, TX 78284

Attn: D. M. Deffenbaugh

Stanford Research Institute

Menlo Park, CA 94025

Attn: A. J. Slemmons

Stearns-Roger

4500 Cherry Creek

Denver, CO 80217

Attn: W. R. Lang

W. B. Stine

317 Monterey Rd., Apt. 22

South Pasadena, CA 91303
Sun Gas Company

Suite 800,2 No. Pk. E

Dallas, TX 75231

Attn: R. C. Clark

Sun-Heet, Inc.

2624 So. Zuni

Englewood, CO 80110

Sundstrand Electric Power

4747 Harrison Avenue

Rockford, IL 61101

Attn: A. W. Adam

Sunpower Systems Corp.

510 S. 52nd St., Suite 101

Tempe, AZ 85281

Attn: W. C. Matlock

Suntec Systems, Inc.

2101 Woodale Drive

St. Paul, MN 55110

Attn: L. W. Rees

Swedlow, Inc.

12122 Western $\Lambda$ venuc

Garden Grove, CA 92645

Attn: E. Nixon

3M-Decorative Products Division 209-2N 3M Center

St. Paul, MN 55101

Attn: B. Benson

3M-produce Development

Energy Control Products

207-1W 3M Center

St. Paul, MN 55101

Attn: J. R. Roche

I'exas 'l'ech University

Dept. of Electrical Engineering

P.O. Box 4709

Lubbock, TX 79409

Attn: J. D. Reichert

TRW, Inc.

Energy Systems Group of TRW, Inc.

One Space Park Bldg. R4, Room 2074

Redondo Beach, CA 90278

Attn: J. M. Cherne 
Toltec Industries, Inc. (20)

40 th and East Main

Clear Lake, IA 50428

Attn: D. Chenault

U.S. Department of Energy (3)

Albuquerque Operations Office

P.O. Box 5400

Albuquerque, NM 87185

Attn: G. N. Pappas

C. B. Quinn

$\mathrm{J}$. Weisiger

U.S. Department of Energy

Division of Energy Storage Systems

Washington, DC 20545

Attn: J. Gahimer

U.S. Department of Energy (8)

Division of Solar Thermal

Energy Systems

Washington, DC 20585

Attn: $W$. W. Auer

G. W. Braun

J. E. Greyerbieh 1

M. U. Gutstein

L. Melamed

J. E. Rannels

F. Wilkins

J. Dollard

U.S. Department of Energy (2)

San Francisco Operations office 1333 Broadway, Wells Fargo Bldg. Oakland, CA 94612

Attn: $R$, W. Hughey

University of Kansas Center for Research, CRINC

2291 Irving Hall Rd.

Lawrence, KS 66045

Attn: R. F. Riordan

University of New Mexico (2)

Department of Mechanical Eng.

Albuquerque, NM 87113

Attn: M. W.Wilden

W. A. Cross
Viking Solar Systems

3223 North Verdugo Road

Glendale, CA 91208

Attn: G. Goranson

Winsmith

Div, of UMC Industries, Inc. Springville, NY 14141

Attn: R. Bhise

Wyle Laboratories

7800 Governor's Drive West

Huntsville, AL 35807

Attn: R. Losey

1520 T. J. Hoban

1530 W. E. Caldes

1550 F. W. Neilson

2320 K. L. Gillespie

2323 C. M. Gabriel

2324 R. S. Pinkham

2326 G. M. Heck

3154-3 R. P. Campbell

3161 J. E. Mitchell

3600 R. W. Hunnicutt

Attn: H. H. Pastorius, 3640

3700 J. C. Strassell

4000 A. Narath

4231 J. H. Renken

4700 J. H. Scott

4710 G. E. Brandvold

4713 B. W. Marshal1

4714 R. P. Stromberg (20)

4715 R. H. Braasch

4718 E. Burgess

4719 D. G. Schueler

4720 V. L. Dugan (100)

4721 Author (20)

4721 J. V. Otts

4722 J. F. Banas

4723 W. P. Schimmel

4725 J. A. Leonard

4730 H. M. Stoller

5510 D. B. Hayes

5513 D. W. Larson

5520 T. B. Lane

5523 R. C. Reuter

5810 R. G. Kepler

5820 R. E. Whan

5830 M. J. Davis

5833 J. L. Jellison 


\section{DISTRIBUTION (Continued)}

$\begin{array}{ll}5840 & \text { N. Magnani } \\ 8266 & \text { E. A. Aas (2) } \\ 8450 & \text { R. C. Wayne } \\ 8451 & \text { C. F. Melius } \\ 8452 & \text { A. C. Skinrood } \\ 8452 & \text { T. Bramlette } \\ 8453 & \text { W. G. Wilson } \\ 3141 & \text { T. L. Werner (5) } \\ 3151 & \text { W. L. Garner (3) } \\ & \text { (Unlimited Release) } \\ & \text { For DOE/TIC } \\ & \text { (Unlimited Release) } \\ 6011 & \text { Patents }\end{array}$




\section{DO NOT MICROFILM
COVER}

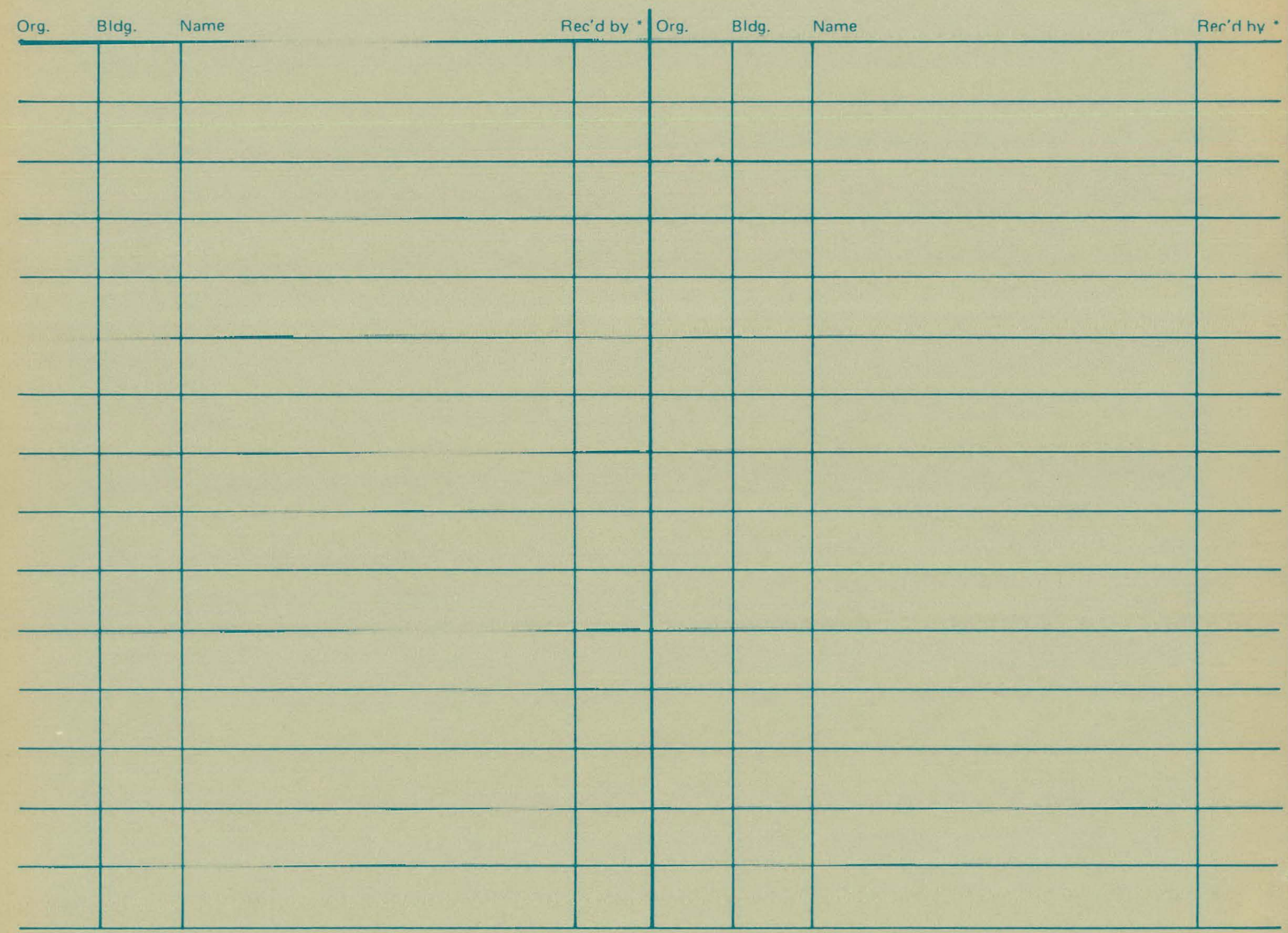

